\title{
新型含吡啶环结构的吡唑肜酯类衍生物的合成与生物活性研究
}

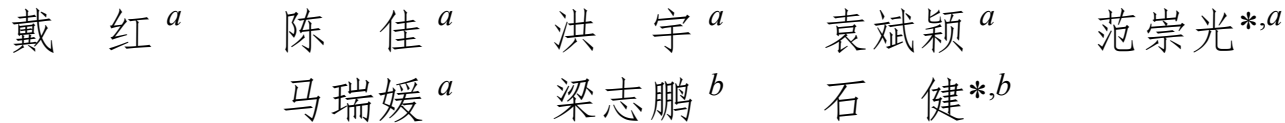 \\ $\left({ }^{a}\right.$ 南通大学化学化工学院 南通 226019) \\ ( $b$ 南通大学分析测试中心 南通 226019)
}

\begin{abstract}
摘要 为了寻找具有较好生物活性的吡唑类衍生物, 通过活性亚结构拼接的方法, 设计制备了一系列未见文献报道的 含取代吡啶结构的吡唑肟醌化合物. 通过 ${ }^{1} \mathrm{H} \mathrm{NMR} 、{ }^{13} \mathrm{C} N \mathrm{NMR}$ 和元素分析对目标化合物的结构进行了表征. 初步的生物 活性测试结果显示, 部分化合物表现出一定的杀虫活性. 在测试浓度为 $500 \mu \mathrm{g} / \mathrm{mL}$ 时, 有 7 个化合物对粘虫的杀灭活性 可达 $60 \% \sim 100 \%, 6$ 个化合物对蜴虫的杀死率可达 $50 \% \sim 90 \%$. 当测试浓度降为 $100 \mu \mathrm{g} / \mathrm{mL}$ 时, 1,3 -二甲基-5-(4-氯苯氧 基)吡唑-4-甲醛- $O$-(2-氯吡啶-3-甲酰基)肜(5f)和 1,3-二甲基-5-(4-甲基苯氧基)吡唑-4-甲醛- $O$-(2-氯吡啶-3-甲酰基)肜(5j) 对䖮虫仍表现出一定的杀虫活性, 其杀死率均为 50\%. 1,3-二甲基-5-(3-氟苯氧基)吡唑-4-甲醛- $O$-(2-氯吡啶-3-甲酰基)肜 (5b) 和 $5 \mathbf{f}$ 在测试浓度为 $500 \mu \mathrm{g} / \mathrm{mL}$ 时对褐飞虫的杀死率均为 $100 \%$. 另外, 1,3-二甲基-5-(4-氟苯氧基)吡唑-4-甲醛- $O$-(2氯吡啶-3-甲酰基)肜(5c)，1３-二甲基-5-(3-氯苯氧基)吡唑-4-甲醛- $O$-(2-氯吡啶-3-甲酰基)肜(5e)，1,3-二甲基-5-(4-三氟甲 氧基苯氧基)吡唑-4-甲醛- $O$-(2-氯吡啶-3-甲酰基)肜(5i)和 $\mathbf{5 j}$ 对人肝癌(HepG2)细胞株显示出明显的抗肿瘤活性, 其 $\mathrm{IC}_{50}$ 值分别为 2.6, 4.6, 1.8 和 $1.1 \mu \mathrm{mol} / \mathrm{L}$.
\end{abstract}

关键词 吡啶; 吡唑; 合成; 生物活性

\section{Synthesis and Bioactivities of Novel Pyrazole Oxime Ester Derivatives Containing Pyridyl Moiety}

\author{
Dai, Hong ${ }^{a}$ \\ Chen, $\mathrm{Jia}^{a}$ \\ Hong, $\mathrm{Yu}^{a}$ \\ Yuan, Binying ${ }^{a}$ \\ Fan, Chongguang ${ }^{*, a}$ \\ Ma, Ruiyuan ${ }^{a} \quad$ Liang, Zhipeng ${ }^{b} \quad$ Shi, Jian*,b \\ $\left({ }^{a}\right.$ College of Chemistry and Chemical Engineering, Nantong University, Nantong 226019) \\ $\left({ }^{b}\right.$ Analysis and Testing Center, Nantong University, Nantong 226019)
}

\begin{abstract}
In order to explore novel pyrazole derivatives with good biological activities, a series of novel pyrazole oxime ester compounds containing pyridyl moiety were designed and synthesized according to the method of active substructure combination. The structures of the target compounds were determined by ${ }^{1} \mathrm{H}$ NMR, ${ }^{13} \mathrm{C}$ NMR and elemental analysis. Preliminary bioassay data indicated that some of the title compounds showed certain insecticidal activities. At a concentration of $500 \mu \mathrm{g} / \mathrm{mL}$, seven compounds exhibited insecticidal activity against Oriental armyworm with $50 \% \sim 90 \%$, and six compounds exhibited insecticidal activity against Aphis medicaginis with 50\% 90\%. When the dosage was lowered to $100 \mu \mathrm{g} / \mathrm{mL}, 1,3$-dimethyl-5(4-chlorophenoxy)pyrazole-4-formyl-O-(2-chloropyridin-3-formyl)oxime (5f) and 1,3-dimethyl-5-(4-methylphenoxy)pyrazole-4-formyl-O-(2-chloropyridin-3-formyl)oxime (5j) were still active against Aphis medicaginis with inhibitory values of $50 \%$ and $50 \%$, respectively. Insecticidal activities against Nilaparvata lugens of 1,3-dimethyl-5-(3-fluorophenoxy)pyrazole4-formyl-O-(2-chloropyridin-3-formyl)oxime (5b) and $\mathbf{5 f}$ were both $100 \%$ at $500 \mu \mathrm{g} / \mathrm{mL}$. Additionally, 1,3-dimethyl-5-(4fluorophenoxy)pyrazole-4-formyl- $O$-(2-chloropyridin-3-formyl)oxime (5c), 1,3-dimethyl-5-(3-chlorophenoxy)-pyrazole-4-formyl-O-(2-chloropyridin-3-formyl)oxime (5e), 1,3-dimethyl-5-(4-trifluoromethoxyphenoxy)pyrazole-4-formyl- $O$-(2-chloropyridin-3-formyl)oxime (5i) and $\mathbf{5 j}$ displayed good anti-tumor activity against HepG2 cells with $\mathrm{IC}_{50}$ values of 2.6, 4.6, 1.8 和 $1.1 \mu \mathrm{mol} / \mathrm{L}$, respectively.
\end{abstract}

* Corresponding authors. E-mail: ntfcg@ntu.edu.cn, gaohbhe2015@aliyun.com

Received January 20, 2017; revised February 21, 2017; published online February 27, 2017.

Project supported by the National Natural Science Foundation of China (No. 21372135), the Research Foundation of the Six People Peak of Jiangsu Province (No. 2013-SWYY-013), and the Science and Technology Project Fund of Nantong City (Nos. CP12013002, MS22015020).

国家自然科学基金(No. 21372135)、江苏省 “六大人才高峰” (No. 2013-SWYY-013)和南通市科技计划(Nos. CP12013002, MS22015020)资助项目. 
Keywords pyridine; pyrazole; synthesis; biological activity

吡唑类衍生物作为含氮杂环体系中的一员, 以其优 良的杀菌、杀虫、杀螨、抗病毒及抗肿瘤等活性而倍受 农药与药物化学工作者的关注 ${ }^{[1 \sim 5]}$. 近年来, 具有多种 农用生物活性的吡唑类化合物被相继开发出来, 如日本 Nihon Nohyaku 公司研制的杀螨剂唑螨酯(Fenpyroximate)具有击倒快、持效时间长、受季节影响小等特点, 可用于防治多种植食性螨 ${ }^{[6,7]}$, 美国 Du Pont 公司开发的 杀虫剂氯虫苯甲酰胺对鳞翅目和半翅目类害虫表现优 异的防效 ${ }^{[8]}$. 许多含吡唑基化合物在医疗保健方面也扮 演着十分重要的角色, 如环氧合酶-2 选择抑制剂塞来考 昔、镇痛药安乃近等 ${ }^{[9,10]}$. 另外, 吡啶环亦为一类重要的 含氮杂环, 吡啶类化合物在农药与医药领域发挥着重要 作用. 吡啶类衍生物因其广谱的生物活性及较好的环境 相容性而引起人们极大的研究兴趣 ${ }^{[11 ~ 17]}$, 有关吡啶类 化合物的分子设计、合成与生物活性研究仍为药物化学 研究的重要领域之一. 此外, 肟酯基在药物分子中也是 一种常见的结构片段 ${ }^{[18 \sim 21]}$, 肟酯单元常常被作为良好 药效团引入到化合物分子中, 以改善其生物活性. 如 Song 等 ${ }^{[22]}$ 报道的含弜酯结构的吡唑酰胺化合物具有优 异的杀虫活性, Liu 等 ${ }^{[23]}$ 合成的含弜酯结构的吡唑类衍 生物显示出良好的抗真菌活性. 鉴于此, 为了进一步寻 找具有较好生物活性的吡唑类化合物, 本研究参照唑螨 酯的结构, 利用活性亚结构拼接原理, 设计并合成了一 系列未见文献报道的新型吡啶结构的吡唑肜酯衍生物 (图 1). 通过 ${ }^{1} \mathrm{H} N M R,{ }^{13} \mathrm{C} N M R$ 和元素分析等方法确证 了目标化合物的结构. 同时对所合成的化合物进行了初 步的生物活性测试, 结果表明部分标题化合物表现出较 好的杀虫效果, 部分目标物显示出较好的抗肿瘤活性. 目标化合物的合成路线如 Scheme 1 所示.
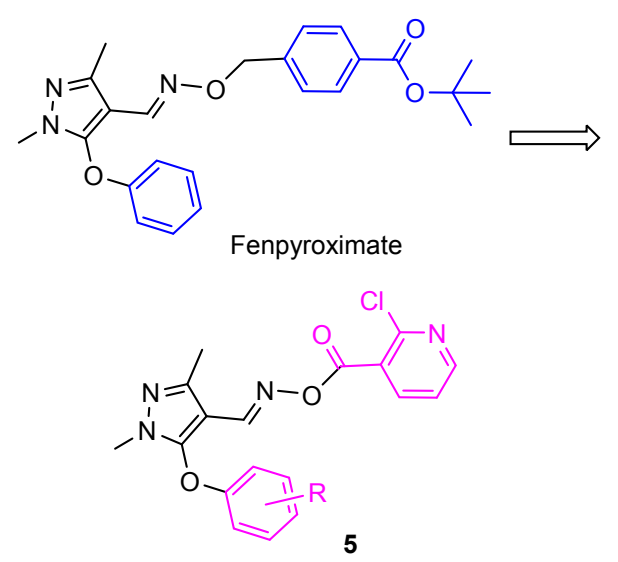

图 1 目标化合物 $\mathbf{5}$ 的分子设计示意图

Figure 1 Design strategy of target compounds 5
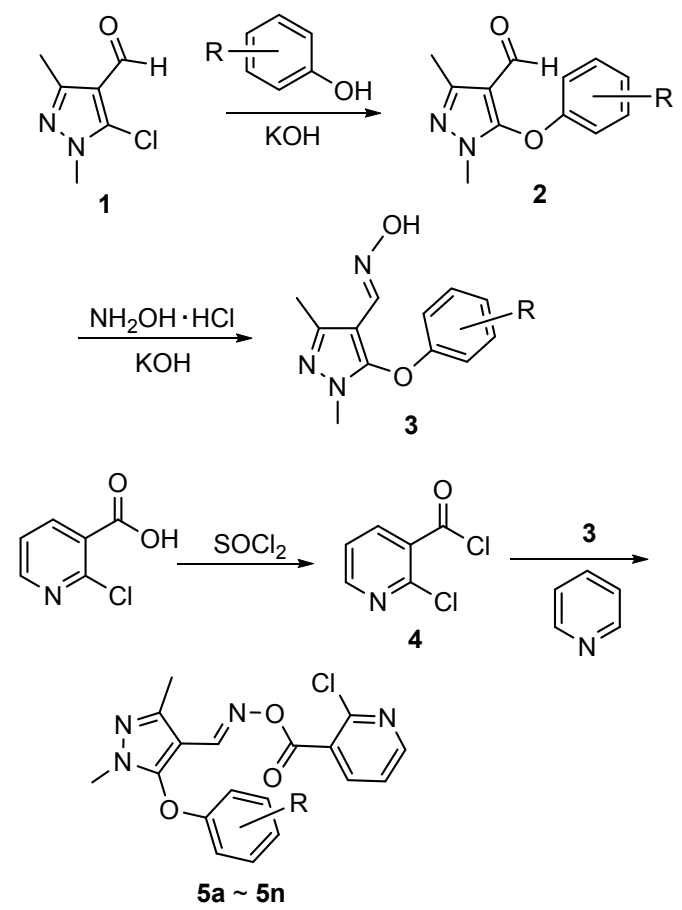

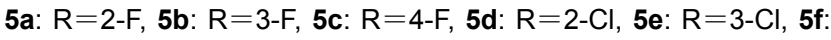
$\mathrm{R}=4-\mathrm{Cl}, 5 \mathbf{g}: \mathrm{R}=2-\mathrm{Br}$, 5h: $\mathrm{R}=2-\mathrm{OCF}_{3}, 5 \mathrm{i}: \mathrm{R}=4-\mathrm{OCF}_{3}, 5 \mathrm{j}: \mathrm{R}=$ $4-\mathrm{CH}_{3}, 5 \mathbf{k}: \mathrm{R}=4-t-\mathrm{C}_{4} \mathrm{H}_{9}, 5 \mathrm{I}: \mathrm{R}=2,3-\mathrm{F}_{2}, 5 \mathbf{m}: \mathrm{R}=2,4-\mathrm{F}_{2}, \mathbf{5 n}: \mathrm{R}=$ $2,4-\mathrm{Cl}_{2}$

图式 1 目标化合物 5 的合成路线

Scheme 1 Synthetic route of title compounds 5

\section{1 结果与讨论}

\section{1 目标化合物的合成}

在目标化合物的合成中, 我们以化合物 $\mathbf{5 i}$ 的合成为 研究对象, 尝试了不同的反应条件对 $5 \mathbf{i}$ 收率的影响. 由 表 1 可以看出, 采用吡啶作缚酸剂, $\mathrm{CH}_{2} \mathrm{Cl}_{2}$ 作溶剂, 室 温反应 $12 \mathrm{~h}$, 是制备目标化合物的较佳方法, 化合物 $\mathbf{5 i}$ 的收率为 $60 \%$, 该方法反应条件温和, 后处理较为简便. 因此，采用该方法顺利地制备了其它目标化合物，并利 用核磁氢谱、碳谱和元素分析等手段对其结构进行了表 征.

表 1 不同反应条件对目标化合物 $5 \mathbf{i}$ 合成收率的影响

Table 1 Effects of reaction conditions on the synthesis of target compound $\mathbf{5 i}$

\begin{tabular}{ccclc}
\hline Entry & Base & Solvent & \multicolumn{1}{c}{ Reaction condition } & Yield/\% \\
\hline 1 & $\mathrm{Et}_{3} \mathrm{~N}$ & Benzene & Reflux, 12 h & 0 \\
2 & $\mathrm{Et}_{3} \mathrm{~N}$ & Toluene & Reflux, 12 h & 0 \\
3 & Pyridine & $\mathrm{CH}_{2} \mathrm{Cl}_{2}$ & Room temperature, 12 h & 60 \\
4 & Pyridine & $\mathrm{CH}_{3} \mathrm{CN}$ & Room temperature, 12 h & 47 \\
5 & Pyridine & $\mathrm{CH}_{2} \mathrm{Cl}_{2}$ & Reflux, 12 h & 53 \\
\hline
\end{tabular}




\section{2 化合物的图谱解析}

我们以目标化合物 $\mathbf{5 f}$ 的核磁氢谱与碳谱数据为例 进行解析. ${ }^{1} \mathrm{H}$ NMR 中 $\delta 8.53$ 处的双重峰对应于吡啶环 上一个氢的吸收峰; $\delta 8.14$ 处的单峰为 $\mathrm{CH}=\mathrm{N}$ 氢的吸收 峰; $\delta 8.09$ 处的双重峰为吡啶上一个氢的吸收峰; $\delta$ $7.31 \sim 7.34$ 多重峰对应于苯环上两个氢和吡啶环上一个 氢的吸收峰; $\delta 6.99$ 处的双重峰为苯环上两个氢的吸收 峰; $\delta 3.63$ 处的单峰为吡唑环 1-位甲基上三个氢的吸收 峰; $\delta 2.49$ 处的单峰为吡唑环 3-位甲基上三个氢的吸收 峰. ${ }^{13} \mathrm{C} \mathrm{NMR}$ 中 $\delta$ 在 162.4 处的峰为与吡啶环相连的 $\mathrm{CO}$ 碳原子的信号峰; $\delta 34.5$ 处的峰为吡唑环 1-位甲基碳原 子的信号峰; $\delta 15.1$ 处的峰为吡唑环 3-位甲基碳原子的 信号峰.

\section{3 化合物的杀虫活性}

目标化合物 $\mathbf{5 a} \sim \mathbf{5 n}$ 对粘虫(Oriental armyworm)、蚜 虫(Aphis medicaginis)和褐飞虫(Nilaparvata lugens)的杀 虫活性测试结果见表 2. 初步的生测结果显示, 部分目 标化合物表现出一定的杀虫活性. 在测试浓度为 500 $\mu \mathrm{g} / \mathrm{mL}$ 时，化合物 5a, 5c, 5e, 5f, 5g, 5h 和 $5 n$ 对粘虫的杀 灭活性分别为 $70 \%, 100 \%, 80 \%, 60 \%, 70 \%, 100 \%$ 和 $100 \%$, 其中化合物 5c, $5 \mathbf{h}$ 和 $\mathbf{5 n}$ 对粘虫的防效与对照药 啶虫丙醚相当; 化合物 $5 b, 5 c, 5 f, 5 \mathrm{~g}, 5 \mathbf{i}$ 和 $5 \mathbf{j}$ 对蚜虫的杀 死率分别为 $50 \%, 50 \%, 90 \%, 50 \%, 60 \%$ 和 $80 \%$, 当测试 浓度降至 $100 \mu \mathrm{g} / \mathrm{mL}$ 时, 化合物 $\mathbf{5 f}$ 和 $\mathbf{5 j}$ 对蚜虫仍具有 中等水平的杀虫活性，其抑制率均为 $50 \%$. 此外，化合 物 $5 \mathbf{b}$ 和 $\mathbf{5 f}$ 在测试浓度为 $500 \mu \mathrm{g} / \mathrm{mL}$ 时对褐飞闽呈现出

表 2 目标化合物 $\mathbf{5 a} \sim \mathbf{5 n}$ 的杀虫活性(死亡率/\%) ${ }^{a}$ Table 2 Insecticidal activities (mortality/\%) of target compounds $5 \mathbf{a} \sim \mathbf{5 n}$

\begin{tabular}{|c|c|c|c|c|c|c|}
\hline \multirow[t]{2}{*}{ Compd. } & \multicolumn{2}{|c|}{$\begin{array}{c}\text { Oriental } \\
\text { armyworm }\end{array}$} & \multicolumn{2}{|c|}{$\begin{array}{c}\text { Aphis } \\
\text { medicaginis }\end{array}$} & \multicolumn{2}{|c|}{$\begin{array}{c}\text { Nilaparvata } \\
\text { lugens }\end{array}$} \\
\hline & 500 & 100 & 500 & 100 & 500 & 100 \\
\hline $5 a$ & 70 & - & 0 & - & 0 & - \\
\hline $5 b$ & 0 & - & 50 & - & 100 & 0 \\
\hline $5 c$ & 100 & 0 & 50 & - & 0 & - \\
\hline $5 d$ & 0 & - & 0 & - & 0 & - \\
\hline $5 e$ & 80 & 0 & 0 & - & 0 & - \\
\hline $5 f$ & 60 & - & 90 & 50 & 100 & 0 \\
\hline $5 g$ & 70 & - & 50 & - & 0 & - \\
\hline $5 \mathrm{~h}$ & 100 & 0 & 0 & - & 0 & - \\
\hline $5 i$ & 0 & - & 60 & - & 0 & - \\
\hline $5 \mathbf{j}$ & 0 & - & 80 & 50 & 0 & - \\
\hline $5 k$ & 0 & - & 0 & - & 0 & - \\
\hline 51 & 40 & - & 0 & - & 0 & - \\
\hline $5 \mathrm{~m}$ & 40 & - & 0 & - & 0 & - \\
\hline $5 n$ & 100 & 0 & 0 & - & 0 & - \\
\hline Pyridalyl & 100 & 100 & - & - & - & - \\
\hline Imidacloprid & - & - & 100 & 100 & 100 & 100 \\
\hline
\end{tabular}

良好的杀灭活性，其抑制率均达 100\%，与对照药吡虫 啉的药效相当. 这为今后继续从事吡唑类化合物的结构 优化与生物活性研究提供了重要的试验数据.

\section{4 化合物的抗肿瘤活性}

目标化合物 $\mathbf{5 a} \sim \mathbf{5 n}$ 对人肝癌 $(H e p G 2)$ 和人胃癌 (SGC7901)细胞株的体外抗肿瘤活性结果如表 3 所示. 从表 3 可以看出, 目标化合物对 SGC7901 癌细胞均未显 示出明显的抑制活性，然而部分目标物对 HepG2 癌细 胞具有较好的抑制作用，但无明显的规律. 4-氟取代物 5c、3-氯取代物 5e、4-三氟甲氧基取代物 5i 和 4-甲基取 代物 5j 对 $\mathrm{HepG} 2$ 细胞的抑制活性相对较好, 其 $\mathrm{IC}_{50}$ 值 分别为 $2.6,4.6,1.8$ 和 $1.1 \mu \mathrm{mol} / \mathrm{L}$, 明显高于对照药 Sorafenib 的活性 $\left(\mathrm{IC}_{50}=16.2 \mu \mathrm{mol} / \mathrm{L}\right)$. 该类型化合物的 结构衍生与抗肿瘤活性研究正在进行中.

表 3 目标化合物 $\mathbf{5 a} \sim \mathbf{5 n}$ 的体外抗肿瘤活性

Table 3 Cytotoxicity of target compounds $\mathbf{5 a} \sim \mathbf{5 n}$

\begin{tabular}{ccc}
\hline \multirow{2}{*}{ Compd. } & \multicolumn{2}{c}{$\mathrm{IC}_{50} /\left(\mu \mathrm{mol} \cdot \mathrm{L}^{-1}\right)$} \\
\cline { 2 - 3 } & HepG2 & SGC7901 \\
$\mathbf{5 a}$ & $>20$ & $>20$ \\
$\mathbf{5 b}$ & $>20$ & $>20$ \\
$\mathbf{5 c}$ & 2.6 & $>20$ \\
$\mathbf{5 d}$ & $>20$ & $>20$ \\
$\mathbf{5 e}$ & 4.6 & $>20$ \\
$\mathbf{5 f}$ & $>20$ & $>20$ \\
$\mathbf{5 g}$ & $>20$ & $>20$ \\
$\mathbf{5 h}$ & $>20$ & $>20$ \\
$\mathbf{5 i}$ & 1.8 & $>20$ \\
$\mathbf{5 j}$ & 1.1 & $>20$ \\
$\mathbf{5 k}$ & $>20$ & $>20$ \\
$\mathbf{5 l}$ & $>20$ & $>20$ \\
$\mathbf{5 m}$ & $>20$ & $>20$ \\
$\mathbf{5 n}$ & $>20$ & 12.1 \\
Sorafenib & 16.2 &
\end{tabular}

\section{2 结论}

通过 2-氯吡啶-3-甲酰基氯与取代吡唑肜的缩合反 应，合成了 14 个新型含吡啶结构的吡唑肟酯类化合物. 初步的生物活性测试结果表明，部分化合物显示出一定 的杀虫活性. 在测试浓度为 $500 \mu \mathrm{g} / \mathrm{mL}$ 时，化合物 $\mathbf{5 a}$, 5c, 5e, 5f， 5g，5h 和 $5 n$ 对粘虫的杀灭效果分别为 70\%, $100 \%, 80 \%, 60 \%, 70 \%, 100 \%$ 和 100\%，化合物 $\mathbf{5 b}, \mathbf{5 c}, \mathbf{5 f}$, $5 \mathrm{~g}, 5 \mathbf{i}$ 和 $5 \mathbf{j}$ 对蚜虫的杀死率分别为 $50 \%, 50 \%, 90 \%, 50 \%$, $60 \%$ 和 $80 \%$ ，化合物 $\mathbf{5 b}$ 和 $\mathbf{5 f}$ 对褐飞闽的抑制活性均为 $100 \%$ ，当测试浓度降至 $100 \mu \mathrm{g} / \mathrm{mL}$ 时，化合物 $\mathbf{5 f}$ 和 $\mathbf{5 j}$ 对蚛虫仍表现出一定的杀虫效果，其杀死率均为 $50 \%$. 此外, 化合物 $\mathbf{5 c}, \mathbf{5 e}, \mathbf{5 i}$ 和 $\mathbf{5 j}$ 对人肝癌(HepG2)细胞株呈 现出较好的抗肿瘤活性, 其 $\mathrm{IC}_{50}$ 值分别为 $2.6,4.6,1.8$ 和 $1.1 \mu \mathrm{mol} / \mathrm{L}$, 高于对照药 Sorafenib 的活性, 具有进一步 
研究的价值.

\section{3 实验部分}

\section{1 仪器与试剂}

X-4 型数字显示熔点测定仪(北京泰克仪器有限公 司), 温度计未经校正; Yanaco-CHN CORDER MT-3 自 动元素分析仪; LCQ-Advantage 型质谱仪; Bruker AM-400 型核磁共振仪, 以 $\mathrm{CDCl}_{3}$ 为溶剂, $\mathrm{TMS}$ 为内标; 柱层析硅胶为 $\mathrm{H}$ 型(青岛海洋化工厂，200～300 目). 所 用试剂均为分析纯. 中间体 1,3-二甲基-5-氯吡唑-4-甲醛 (1)按照文献[24]方法制备，1,3-二甲基-5-取代苯氧基吡 唑-4-甲醛(2)和 1,3-二甲基-5-取代苯氧基吡唑-4-甲醛肟 (3)按照文献[25, 26]方法制备.

\subsection{2-氯吡啶-3-甲酰基氯(4)的合成}

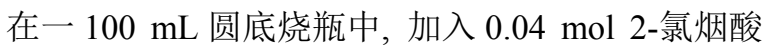
及 $0.2 \mathrm{~mol}$ 二氯亚砜, 室温搅拌下, 向其中加入几滴 $N, N$-二甲基甲酰胺(DMF), 加热回流 $5 \mathrm{~h}$, 冷却至室温, 减压蒸除溶剂, 得到相应的中间体 4 , 产品未经纯化可 直接用于下一步反应.

\section{3 目标化合物 5 的合成}

在一 $100 \mathrm{~mL}$ 圆底烧瓶中, 加入 $3 \mathrm{mmol}$ 肜中间体 $\mathbf{3}$ 、 $3 \mathrm{~mL}$ 吡啶及 $25 \mathrm{~mL}$ 二氯甲烷，冰浴下搅拌，向其中慢慢 滴加 $3.6 \mathrm{mmol} 2$-氯吡啶-3-甲酰基氯(4)的二氯甲烷溶液 (15 mL). 滴毕, 室温摚拌 $10 \sim 16 \mathrm{~h}$. 减压蒸去溶剂, 向 残余物中加入 $50 \mathrm{~mL}$ 二氯甲烷, 用饱和食盐水洗涤, 无 水硫酸钠干燥, 抽滤, 脱溶, 所得残余物以石油醚/乙酸 乙酯 $(V: V=20: 1)$ 为洗脱剂进行柱层析分离, 得到目 标化合物 $\mathbf{5 a} \sim \mathbf{5 n}$.

1,3-二甲基-5-(2-氟苯氧基)吡唑-4-甲醛- $O$-(2-氯吡 啶-3-甲酰基)肜(5a)：白色固体，产率 53\%. m.p. 105 $106{ }^{\circ} \mathrm{C} ;{ }^{1} \mathrm{H}$ NMR $\left(400 \mathrm{MHz}, \mathrm{CDCl}_{3}\right) \delta: 8.54$ (d, $J=4.0$ $\mathrm{Hz}, 1 \mathrm{H}, \mathrm{Py}-\mathrm{H}), 8.14$ (s, $1 \mathrm{H}, \mathrm{CH}=\mathrm{N}), 8.10(\mathrm{~d}, J=8.0 \mathrm{~Hz}$, 1H, Рy-H), $7.33 \sim 7.36$ (m, 1H, Рy-H), $7.08 \sim 7.25$ (m, 3H, Ar-H), $6.87 \sim 6.91(\mathrm{~m}, 1 \mathrm{H}, \mathrm{Ar}-\mathrm{H}), 3.69\left(\mathrm{~s}, 3 \mathrm{H}, \mathrm{N}-\mathrm{CH}_{3}\right)$, $2.49\left(\mathrm{~s}, 3 \mathrm{H}, \mathrm{CH}_{3}\right) ;{ }^{13} \mathrm{C}$ NMR $\left(100 \mathrm{MHz}, \mathrm{CDCl}_{3}\right) \delta: 162.4$, $153.3,151.9,150.8,149.7,148.9,148.1,144.0,140.0$, $126.4,125.4,124.9,122.1,117.7,117.4,98.0,34.5,15.2$. Anal. calcd for $\mathrm{C}_{18} \mathrm{H}_{14} \mathrm{ClFN}_{4} \mathrm{O}_{3}$ : C 55.61, H 3.63, N 14.41; found $\mathrm{C} 55.73, \mathrm{H} 3.53, \mathrm{~N} 14.28$.

1,3-二甲基-5-(3-氟苯氧基)吡唑-4-甲醛- $O$-(2-氯吡 啶-3-甲酰基)肜(5b): 白色固体, 产率 58\%. m.p. 104 $106{ }^{\circ} \mathrm{C} ;{ }^{1} \mathrm{H}$ NMR (400 MHz, $\left.\mathrm{CDCl}_{3}\right) \delta: 8.53$ (d, $J=4.0$ $\mathrm{Hz}, 1 \mathrm{H}, \mathrm{Py}-\mathrm{H}), 8.16(\mathrm{~s}, 1 \mathrm{H}, \mathrm{CH}=\mathrm{N}), 8.09(\mathrm{~d}, J=8.0 \mathrm{~Hz}$, 1H, Py-H), $7.29 \sim 7.35$ (m, 2H, Py-H and Ar-H), 6.85 $6.89(\mathrm{~m}, 1 \mathrm{H}, \mathrm{Ar}-\mathrm{H}), 6.68 \sim 6.73(\mathrm{~m}, 2 \mathrm{H}, \mathrm{Ar}-\mathrm{H}), 3.64(\mathrm{~s}$, $\left.3 \mathrm{H}, \mathrm{N}-\mathrm{CH}_{3}\right), 2.51\left(\mathrm{~s}, 3 \mathrm{H}, \mathrm{CH}_{3}\right) ;{ }^{13} \mathrm{C} \mathrm{NMR}(100 \mathrm{MHz}$, $\left.\mathrm{CDCl}_{3}\right) \delta: 164.8,162.4,157.5,151.9,149.7,149.2,148.9$, $148.2,139.9,131.3,126.4,122.1,111.5,111.0,104.0$, 98.7, 34.5, 15.1. Anal. calcd for $\mathrm{C}_{18} \mathrm{H}_{14} \mathrm{ClFN}_{4} \mathrm{O}_{3}$ : C 55.61, H 3.63, N 14.41; found C 55.50, H 3.77, N 14.53.

1,3-二甲基-5-(4-氟苯氧基)吡唑-4-甲醛- $O$-(2-氯吡 啶-3-甲酰基)肜(5c)：白色固体，产率 63\%. m.p. 117 $119{ }^{\circ} \mathrm{C} ;{ }^{1} \mathrm{H}$ NMR $\left(400 \mathrm{MHz}, \mathrm{CDCl}_{3}\right) \delta: 8.53$ (d, $J=4.0$ $\mathrm{Hz}, 1 \mathrm{H}, \mathrm{Py}-\mathrm{H}), 8.13(\mathrm{~s}, 1 \mathrm{H}, \mathrm{CH}=\mathrm{N}), 8.10(\mathrm{~d}, J=8.0 \mathrm{~Hz}$, $1 \mathrm{H}, \mathrm{Py}-\mathrm{H}), 7.32 \sim 7.35$ (m, 1H, Py-H), $6.91 \sim 7.07(\mathrm{~m}, 4 \mathrm{H}$, Ar-H), 3.64 (s, 3H, N-CH $), 2.49\left(\mathrm{~s}, 3 \mathrm{H}, \mathrm{CH}_{3}\right) ;{ }^{13} \mathrm{C} \mathrm{NMR}$ $\left(100 \mathrm{MHz}, \mathrm{CDCl}_{3}\right) \delta: 162.4,160.0,158.1,152.5,151.9$, $149.9,149.7,149.0,148.2,139.9,126.4,122.1,117.0$, $116.9,116.7,98.2,34.5,15.2$. Anal. calcd for $\mathrm{C}_{18} \mathrm{H}_{14} \mathrm{Cl}-$ $\mathrm{FN}_{4} \mathrm{O}_{3}$ : C 55.61, H 3.63, N 14.41; found C 55.75, H 3.56, $\mathrm{N} 14.50$.

1,3-二甲基-5-(2-氯苯氧基)吡唑-4-甲醛- $O$-(2-氯吡 啶-3-甲酰基)肜(5d)：白色固体，产率 55\%. m.p. 124 $126{ }^{\circ} \mathrm{C} ;{ }^{1} \mathrm{H}$ NMR $\left(400 \mathrm{MHz}, \mathrm{CDCl}_{3}\right) \delta: 8.53$ (d, $J=4.0$ $\mathrm{Hz}, 1 \mathrm{H}, \mathrm{Py}-\mathrm{H}), 8.09$ (d, $J=8.0 \mathrm{~Hz}, 2 \mathrm{H}, \mathrm{Py}-\mathrm{H}$ and $\mathrm{CH}=\mathrm{N})$, 7.49 (d, $J=8.0 \mathrm{~Hz}, 1 \mathrm{H}$, Ar-H), $7.31 \sim 7.34$ (m, 1H, Py-H), $7.20 \sim 7.25(\mathrm{~m}, 1 \mathrm{H}, \operatorname{Ar}-\mathrm{H}), 7.10 \sim 7.14(\mathrm{~m}, 1 \mathrm{H}, \mathrm{Ar}-\mathrm{H})$, $6.77 \sim 6.80(\mathrm{~m}, 1 \mathrm{H}, \mathrm{Ar}-\mathrm{H}), 3.66\left(\mathrm{~s}, 3 \mathrm{H}, \mathrm{N}-\mathrm{CH}_{3}\right), 2.50(\mathrm{~s}$, $\left.3 \mathrm{H}, \mathrm{CH}_{3}\right) ;{ }^{13} \mathrm{C}$ NMR $\left(100 \mathrm{MHz}, \mathrm{CDCl}_{3}\right) \delta: 162.4,152.0$, $151.9,149.7,149.6,149.0,148.1,139.9,131.3,128.3$, $126.5,125.4,123.1,122.1,116.2$, 98.3, 34.5, 15.3. Anal. calcd for $\mathrm{C}_{18} \mathrm{H}_{14} \mathrm{Cl}_{2} \mathrm{~N}_{4} \mathrm{O}_{3}$ : C 53.35, H 3.48, N 13.83; found C 53.21, H 3.61, N 13.97.

1,3-二甲基-5-(3-氯苯氧基)吡唑-4-甲醛- $O$-(2-氯吡 啶-3-甲酰基)肜(5e)：淡黄色固体，产率 59\%. m.p. 131 $133{ }^{\circ} \mathrm{C} ;{ }^{1} \mathrm{H}$ NMR (400 MHz, $\mathrm{CDCl}_{3}$ ) $\delta: 8.53$ (d, $J=4.0$ $\mathrm{Hz}, 1 \mathrm{H}, \mathrm{Py}-\mathrm{H}), 8.15$ (s, $1 \mathrm{H}, \mathrm{CH}=\mathrm{N}), 8.09(\mathrm{~d}, J=8.0 \mathrm{~Hz}$, 1H, Py-H), $7.29 \sim 7.34$ (m, 2H, Py-H and Ar-H), 7.14 (d, $J=8.0 \mathrm{~Hz}, 1 \mathrm{H}, \mathrm{Ar}-\mathrm{H}), 6.96(\mathrm{~s}, 1 \mathrm{H}, \mathrm{Ar}-\mathrm{H}), 6.81 \sim 6.84(\mathrm{~m}$, $1 \mathrm{H}, \mathrm{Ar}-\mathrm{H}), 3.64\left(\mathrm{~s}, 3 \mathrm{H}, \mathrm{N}-\mathrm{CH}_{3}\right), 2.50\left(\mathrm{~s}, 3 \mathrm{H}, \mathrm{CH}_{3}\right) ;{ }^{13} \mathrm{C}$ NMR $\left(100 \mathrm{MHz}, \mathrm{CDCl}_{3}\right) \delta: 162.4,157.0,151.9,149.7$, $149.1,148.9,148.3,139.9,135.8,131.1,126.4,124.6$, 122.1, 116.2, 113.6, 98.7, 34.6, 15.1. Anal. calcd for $\mathrm{C}_{18} \mathrm{H}_{14} \mathrm{Cl}_{2} \mathrm{~N}_{4} \mathrm{O}_{3}$ : C 53.35, H 3.48, N 13.83; found C 53.49, $\mathrm{H} 3.34, \mathrm{~N} 13.94$.

1,3-二甲基-5-(4-氯苯氧基)吡唑-4-甲醛- $O$-(2-氯吡 啶-3-甲酰基)肜(5f)：白色固体，产率 66\%. m.p. 110 $112{ }^{\circ} \mathrm{C} ;{ }^{1} \mathrm{H}$ NMR (400 MHz, $\mathrm{CDCl}_{3}$ ) $\delta: 8.53$ (d, $J=4.0$ $\mathrm{Hz}, 1 \mathrm{H}, \mathrm{Py}-\mathrm{H}), 8.14(\mathrm{~s}, 1 \mathrm{H}, \mathrm{CH}=\mathrm{N}), 8.09(\mathrm{~d}, J=8.0 \mathrm{~Hz}$, 
1H, Py-H), $7.31 \sim 7.34$ (m, 3H, Py-H and Ar-H), 6.99 (d, $J=8.0 \mathrm{~Hz}, 2 \mathrm{H}, \mathrm{Ar}-\mathrm{H}$ ), 3.63 (s, 3H, N-CH ${ }_{3}$ ), 2.49 (s, 3H, $\left.\mathrm{CH}_{3}\right) ;{ }^{13} \mathrm{C}$ NMR (100 MHz, $\left.\mathrm{CDCl}_{3}\right) \delta: 162.4,155.1,152.0$, $149.7,149.4,148.9,148.2,140.0,130.2,129.5,126.3$, 122.1, 116.8, 98.4, 34.5, 15.1. Anal. calcd for $\mathrm{C}_{18} \mathrm{H}_{14} \mathrm{Cl}_{2}$ $\mathrm{N}_{4} \mathrm{O}_{3}$ : C 53.35, H 3.48, N 13.83; found C 53.26, H 3.62, N 13.70 .

1,3-二甲基-5-(2-溴苯氧基)吡唑-4-甲醛- $O$-(2-氯吡 啶-3-甲酰基)肜 (5g)：白色固体，产率 52\%. m.p. 134 $136{ }^{\circ} \mathrm{C} ;{ }^{1} \mathrm{H}$ NMR $\left(400 \mathrm{MHz}, \mathrm{CDCl}_{3}\right) \delta: 8.52$ (d, $J=4.0$ $\mathrm{Hz}, 1 \mathrm{H}, \mathrm{Py}-\mathrm{H}), 8.09$ (d, $J=8.0 \mathrm{~Hz}, 2 \mathrm{H}, \mathrm{Py}-\mathrm{H}$ and $\mathrm{CH}=\mathrm{N}$ ), $7.66(\mathrm{~d}, J=8.0 \mathrm{~Hz}, 1 \mathrm{H}, \mathrm{Ar}-\mathrm{H}), 7.25 \sim 7.34(\mathrm{~m}, 2 \mathrm{H}, \mathrm{Py}-\mathrm{H}$ and Ar-H), $7.04 \sim 7.08(\mathrm{~m}, 1 \mathrm{H}, \mathrm{Ar}-\mathrm{H}), 6.75(\mathrm{~d}, J=8.0 \mathrm{~Hz}$, $1 \mathrm{H}, \mathrm{Ar}-\mathrm{H}), 3.65$ (s, 3H, N-CH 3$), 2.51\left(\mathrm{~s}, 3 \mathrm{H}, \mathrm{CH}_{3}\right),{ }^{13} \mathrm{C}$ NMR $\left(100 \mathrm{MHz}, \mathrm{CDCl}_{3}\right) \delta: 162.4,153.1,151.9,149.7$, $149.6,149.0,148.1,139.9,134.4,129.1,126.5,125.8$, 122.1, 116.0, 111.7, 98.4, 34.6, 15.3. Anal. calcd for $\mathrm{C}_{18^{-}}$ $\mathrm{H}_{14} \mathrm{BrClN}_{4} \mathrm{O}_{3}: \mathrm{C} 48.08, \mathrm{H} 3.14, \mathrm{~N} 12.46$; found C 48.01, $\mathrm{H}$ 3.28, N 12.60 .

1,3-二甲基-5-(2-三氟甲氧基苯氧基)吡唑-4-甲醛$O$-(2-氯吡啶-3-甲酰基)肜 (5h): 白色固体, 产率 51\%. m.p. $106 \sim 108{ }^{\circ} \mathrm{C} ;{ }^{1} \mathrm{H}$ NMR (400 MHz, $\mathrm{CDCl}_{3}$ ) $\delta: 8.53$ (d, $J=4.0 \mathrm{~Hz}, 1 \mathrm{H}, \mathrm{Py}-\mathrm{H}), 8.10$ (d, $J=8.0 \mathrm{~Hz}, 2 \mathrm{H}, \mathrm{Py}-\mathrm{H}$ and $\mathrm{CH}=\mathrm{N}), 7.40(\mathrm{~d}, J=8.0 \mathrm{~Hz}, 1 \mathrm{H}, \mathrm{Ar}-\mathrm{H}), 7.32 \sim 7.36$ (m, 1H, Py-H), $7.18 \sim 7.29$ (m, 2H, Ar-H), $6.80(\mathrm{~d}, J=8.0$ $\mathrm{Hz}, 1 \mathrm{H}, \mathrm{Ar}-\mathrm{H}), 3.65$ (s, $\left.3 \mathrm{H}, \mathrm{N}-\mathrm{CH}_{3}\right), 2.52\left(\mathrm{~s}, 3 \mathrm{H}, \mathrm{CH}_{3}\right) ;{ }^{13} \mathrm{C}$ NMR $\left(100 \mathrm{MHz}, \mathrm{CDCl}_{3}\right) \delta: 162.4,151.9,149.7,148.9$, $148.8,148.6,148.2,139.9,128.5,126.4,124.9,124.0$, 122.1, 116.0, 98.5, 34.4, 15.3. Anal. calcd for $\mathrm{C}_{19} \mathrm{H}_{14} \mathrm{Cl}-$ $\mathrm{F}_{3} \mathrm{~N}_{4} \mathrm{O}_{4}$ : C 50.18, H 3.10, N 12.32; found C 50.31, H 3.01, $\mathrm{N} 12.18$.

1,3-二甲基-5-(4-三氟甲氧基苯氧基)吡唑-4-甲醛$O$-(2-氯吡啶-3-甲酰基)肜(5i): 淡黄色固体, 产率 $60 \%$. m.p. $93 \sim 95{ }^{\circ} \mathrm{C} ;{ }^{1} \mathrm{H}$ NMR (400 MHz, $\left.\mathrm{CDCl}_{3}\right) \delta: 8.53$ (d, $J=4.0 \mathrm{~Hz}, 1 \mathrm{H}, \mathrm{Py}-\mathrm{H}), 8.15(\mathrm{~s}, 1 \mathrm{H}, \mathrm{CH}=\mathrm{N}), 8.08(\mathrm{~d}, J=$ $8.0 \mathrm{~Hz}, 1 \mathrm{H}, \mathrm{Py}-\mathrm{H}), 7.31 \sim 7.34$ (m, 1H, Py-H), 7.22 (d, $J=$ $8.0 \mathrm{~Hz}, 2 \mathrm{H}, \mathrm{Ar}-\mathrm{H}$ ), 6.97 (d, $J=8.0 \mathrm{~Hz}, 2 \mathrm{H}, \mathrm{Ar}-\mathrm{H}), 3.65$ (s, $\left.3 \mathrm{H}, \mathrm{N}-\mathrm{CH}_{3}\right), 2.49\left(\mathrm{~s}, 3 \mathrm{H}, \mathrm{CH}_{3}\right) ;{ }^{13} \mathrm{C}$ NMR $(100 \mathrm{MHz}$, $\left.\mathrm{CDCl}_{3}\right) \delta: 162.3,154.8,152.0,149.7,149.3,148.8,148.3$, 145.3, 139.9, 126.4, 123.1, 122.1, 116.7, 98.5, 34.5, 15.0. Anal. calcd for $\mathrm{C}_{19} \mathrm{H}_{14} \mathrm{ClF}_{3} \mathrm{~N}_{4} \mathrm{O}_{4}$ : C 50.18, H 3.10, N 12.32; found $\mathrm{C} 50.05, \mathrm{H} 3.24, \mathrm{~N} 12.46$.

1,3-二甲基-5-(4-甲基苯氧基)吡唑-4-甲醛- $O$-(2-氯 吡啶-3-甲酰基)肜(5j)：白色固体, 产率 63\%. m.p. 64 $66{ }^{\circ} \mathrm{C} ;{ }^{1} \mathrm{H}$ NMR $\left(400 \mathrm{MHz}, \mathrm{CDCl}_{3}\right) \delta: 8.52(\mathrm{~d}, J=4.0 \mathrm{~Hz}$,
1H, Py-H), $8.12(\mathrm{~s}, 1 \mathrm{H}, \mathrm{CH}=\mathrm{N}), 8.10(\mathrm{~d}, J=8.0 \mathrm{~Hz}, 1 \mathrm{H}$, Py-H), $7.32 \sim 7.35$ (m, 1H, Py-H), 7.14 (d, $J=8.0 \mathrm{~Hz}, 2 \mathrm{H}$, Ar-H), 6.84 (d, $J=8.0 \mathrm{~Hz}, 2 \mathrm{H}, \mathrm{Ar}-\mathrm{H}), 3.62$ (s, 3H, N-CH ${ }_{3}$, $2.50\left(\mathrm{~s}, 3 \mathrm{H}, \mathrm{CH}_{3}\right), 2.32\left(\mathrm{~s}, 3 \mathrm{H}, \mathrm{Ar}-\mathrm{CH}_{3}\right) ;{ }^{13} \mathrm{C}$ NMR $(100$ $\left.\mathrm{MHz}, \mathrm{CDCl}_{3}\right) \delta: 162.5,154.5,151.9,150.5,149.7,149.4$, $148.0,140.0,134.0,130.7,126.5,122.1,117.9,115.5$, 98.2, 34.4, 20.6, 15.4. Anal. calcd for $\mathrm{C}_{19} \mathrm{H}_{17} \mathrm{ClN}_{4} \mathrm{O}_{3}$ : C 59.30, H 4.45, N 14.56; found C 59.17, H 4.59, N 14.43.

1,3-二甲基-5-(4-叔丁基苯氧基)吡唑-4-甲醛- $O$-(2氯吡啶-3-甲酰基)肜 $(\mathbf{5 k})$ : 淡黄色固体, 产率 61\%. m.p. $124 \sim 126{ }^{\circ} \mathrm{C} ;{ }^{1} \mathrm{H}$ NMR $\left(400 \mathrm{MHz}, \mathrm{CDCl}_{3}\right) \delta: 8.52(\mathrm{~d}, J=$ $4.0 \mathrm{~Hz}, 1 \mathrm{H}, \mathrm{Py}-\mathrm{H}), 8.11$ (s, $1 \mathrm{H}, \mathrm{CH}=\mathrm{N}), 8.09$ (d, $J=8.0$ $\mathrm{Hz}, 1 \mathrm{H}, \mathrm{Py}-\mathrm{H}), 7.36$ (d, $J=12.0 \mathrm{~Hz}, 2 \mathrm{H}$, Ar-H), $7.30 \sim$ 7.33 (m, 1H, Py-H), 6.87 (d, J=12.0 Hz, 2H, Ar-H), 3.63 (s, $\left.3 \mathrm{H}, \mathrm{N}-\mathrm{CH}_{3}\right), 2.51\left(\mathrm{~s}, 3 \mathrm{H}, \mathrm{CH}_{3}\right), 1.30\left(\mathrm{~s}, 9 \mathrm{H}, t-\mathrm{C}_{4} \mathrm{H}_{9}\right) ;{ }^{13} \mathrm{C}$ NMR $\left(100 \mathrm{MHz}, \mathrm{CDCl}_{3}\right) \delta: 162.5,154.4,151.9,150.5$, $149.7,149.5,148.0,147.4,139.9,127.1,126.5,122.1$, 115.1, 98.3, 34.5, 34.4, 31.4, 15.4. Anal. calcd for $\mathrm{C}_{22} \mathrm{H}_{23}-$ $\mathrm{ClN}_{4} \mathrm{O}_{3}$ : C 61.90, H 5.43, N 13.12; found C 61.99, H 5.31, $\mathrm{N} 13.01$.

1,3-二甲基-5-(2,3-二氟苯氧基)吡唑-4-甲醛- $O$-(2-氯 吡啶-3-甲酰基)肜(5I)：白色固体，产率 52\%. m.p. 122 $123{ }^{\circ} \mathrm{C} ;{ }^{1} \mathrm{H}$ NMR $\left(400 \mathrm{MHz}, \mathrm{CDCl}_{3}\right) \delta: 8.53(\mathrm{~d}, J=4.0$ $\mathrm{Hz}, 1 \mathrm{H}, \mathrm{Py}-\mathrm{H}), 8.16(\mathrm{~s}, 1 \mathrm{H}, \mathrm{CH}=\mathrm{N}), 8.09(\mathrm{~d}, J=8.0 \mathrm{~Hz}$, 1H, Py-H), $7.32 \sim 7.35$ (m, 1H, Py-H), $6.98 \sim 7.03(\mathrm{~m}, 2 \mathrm{H}$, Ar-H), $6.62 \sim 6.65(\mathrm{~m}, 1 \mathrm{H}, \mathrm{Ar}-\mathrm{H}), 3.69\left(\mathrm{~s}, 3 \mathrm{H}, \mathrm{N}-\mathrm{CH}_{3}\right)$, $2.47\left(\mathrm{~s}, 3 \mathrm{H}, \mathrm{CH}_{3}\right) ;{ }^{13} \mathrm{C} \mathrm{NMR}\left(100 \mathrm{MHz}, \mathrm{CDCl}_{3}\right) \delta: 162.3$, $152.0,149.7,148.9,148.6,148.3,145.5,139.9,126.4$, 123.9, 123.7, 122.1, 113.1, 112.9, 111.9, 98.3, 34.5, 14.9. Anal. calcd for $\mathrm{C}_{18} \mathrm{H}_{13} \mathrm{ClF}_{2} \mathrm{~N}_{4} \mathrm{O}_{3}$ : C 53.15, H 3.22, N 13.77; found $\mathrm{C} 53.02, \mathrm{H} 3.36, \mathrm{~N} 13.89$.

1,3-二甲基-5-(2,4-二氟苯氧基)吡唑-4-甲醛- $O$-(2-氯 吡啶-3-甲酰基)肜 (5m): 白色固体，产率 54\%. m.p. $113 \sim 114{ }^{\circ} \mathrm{C} ;{ }^{1} \mathrm{H}$ NMR $\left(400 \mathrm{MHz}, \mathrm{CDCl}_{3}\right) \delta: 8.54$ (d, $J=$ $4.0 \mathrm{~Hz}, 1 \mathrm{H}, \mathrm{Py}-\mathrm{H}), 8.12$ (s, $1 \mathrm{H}, \mathrm{CH}=\mathrm{N}), 8.09$ (d, $J=8.0$ $\mathrm{Hz}, 1 \mathrm{H}, \mathrm{Py}-\mathrm{H}), 7.32 \sim 7.35$ (m, 1H, Py-H), $6.91 \sim 7.01(\mathrm{~m}$, $2 \mathrm{H}$, Ar-H), $6.81 \sim 6.86(\mathrm{~m}, 1 \mathrm{H}, \operatorname{Ar}-\mathrm{H}), 3.71(\mathrm{~s}, 3 \mathrm{H}$, $\left.\mathrm{N}-\mathrm{CH}_{3}\right), 2.45$ (s, $\left.3 \mathrm{H}, \mathrm{CH}_{3}\right),{ }^{13} \mathrm{C} \mathrm{NMR}\left(100 \mathrm{MHz}, \mathrm{CDCl}_{3}\right) \delta$ : $162.3,152.0,149.7,149.6,148.6,148.3,140.0,126.4$, 122.1, 118.5, 118.4, 111.6, 111.4, 106.2, 106.0, 97.7, 34.5, 14.8. Anal. calcd for $\mathrm{C}_{18} \mathrm{H}_{13} \mathrm{ClF}_{2} \mathrm{~N}_{4} \mathrm{O}_{3}$ : C 53.15, H 3.22, N 13.77; found C 53.05, H 3.08, N 13.90 .

1,3-二甲基-5-(2,4-二氯苯氧基)吡唑-4-甲醛- $O$-(2-氯 吡啶-3-甲酰基)肪(5n)：白色固体，产率 50\%. m.p. 133 $135{ }^{\circ} \mathrm{C} ;{ }^{1} \mathrm{H}$ NMR $\left(400 \mathrm{MHz}, \mathrm{CDCl}_{3}\right) \delta: 8.53(\mathrm{~d}, J=4.0$ 
$\mathrm{Hz}, 1 \mathrm{H}, \mathrm{Py}-\mathrm{H}), 8.12$ (s, 1H, CH=N), $8.10(\mathrm{~d}, J=8.0 \mathrm{~Hz}$, 1H, Py-H), 7.50 (s, 1H, Ar-H), $7.32 \sim 7.35$ (m, 1H, Py-H), 7.19 (d, $J=8.0 \mathrm{~Hz}, 1 \mathrm{H}, \operatorname{Ar}-\mathrm{H}), 6.71$ (d, $J=8.0 \mathrm{~Hz}, 1 \mathrm{H}$, Ar-H), 3.66 (s, 3H, N-CH $), 2.49$ (s, 3H, $\left.\mathrm{CH}_{3}\right) ;{ }^{13} \mathrm{C} \mathrm{NMR}$ $\left(100 \mathrm{MHz}, \mathrm{CDCl}_{3}\right) \delta: 162.3,152.0,150.8,149.7,148.9$, $148.6,148.3,140.0,131.0,130.0,128.3,126.3,123.9$, 122.1, 116.7, 98.4, 34.5, 15.1. Anal. calcd for $\mathrm{C}_{18} \mathrm{H}_{13^{-}}$ $\mathrm{Cl}_{3} \mathrm{~N}_{4} \mathrm{O}_{3}$ : C 49.17, $\mathrm{H} 2.98, \mathrm{~N} 12.74$; found C 49.31, H 2.85, $\mathrm{N} 12.86$.

\section{4 生物活性测试}

用分析天平称取一定质量的原药, 用含吐温-80 乳 化剂的 DMF 溶解配制成 $1.0 \%$ 母液, 然后用蒸馏水稀释 备用. 每个处理 3 次重复, 设空白对照.

杀虫活性测试所选昆虫分别为粘虫 (Oriental armyworm)、蚜虫(Aphis medicaginis)和褐飞虫(Nilaparvata lugens). 对照药剂分别为啶虫丙醚(Pyridalyl)和吡 虫啉(Imidacloprid).

蚜虫和褐飞闽: 采用喷雾法. 首先, 分别将接有蚜 虫的蚕豆叶片和接有褐飞虫的水稻苗于 Potter 喷雾塔下 喷雾处理, 处理后蚜虫置于 $20 \sim 22{ }^{\circ} \mathrm{C}$ 观察室内培养, 褐飞闽置于 $24 \sim 27{ }^{\circ} \mathrm{C}$ 观察室内培养, 调查药后 $2 \mathrm{~d}$ 的 死活虫数, 并进行统计分析.

粘虫: 采用浸叶碟法. 首先, 将适量玉米叶在配好 的药液中充分浸润后自然阴干, 放入垫有滤纸的培养血 中, 接粘虫 3 龄中期幼虫 10 头/血, 置于 $24 \sim 27{ }^{\circ} \mathrm{C}$ 观察 室内培养, 调查药后 $2 \mathrm{~d}$ 的死活虫数, 并进行统计分析.

抗肿瘤活性测试所用细胞株分别为人肝癌(HepG2) 和人胃癌(SGC7901)细胞株. 阳性对照药为索拉菲尼 (Sorafenib). 采用噻唑蓝(MTT)法测定了目标化合物的 体外抗肿瘤活性, 具体方法参照文献[27, 28].

\section{辅助材料(Supporting Information) 化合物 $\mathbf{5 a} \sim 5 \mathbf{n}$ 的 ${ }^{1} \mathrm{H}$ NMR 和 ${ }^{13} \mathrm{C}$ NMR 图谱. 这些材料可以免费从本刊 网站(http://sioc-journal.cn/)上下载.}

\section{Referenes}

[1] Li, Y.; Zhang, H. Q.; Liu, J.; Yang, X. P.; Liu, Z. J. J. Agric. Food Chem. 2006, 54, 3636.

[2] Dai, H.; Li, Y. Q.; Du, D.; Qin, X.; Zhang, X.; Yu, H. B.; Fang, J. X. J. Agric. Food Chem. 2008, 56, 10805.

[3] Hamaguchi, H.; Kajihara, O.; Katoh, M. J. Pestic. Sci. 1995, 20, 173.

[4] Motoba, K.; Nishizawa, H.; Suzuki, T.; Hamaguchi, H.; Uchida, M.; Funayama, S. Pestic. Biochem. Physiol. 2000, 67, 73.

[5] Park, H. J.; Lee, K.; Park, S. J.; Ahn, B.; Lee, J. C.; Cho, H. Y.; Lee, K. I. Bioorg. Med. Chem. Lett. 2005, 15, 3307.

[6] Hamaguchi, H.; Kajihara, O.; Katoh, M. J. Pestic. Sci. 1995, 20, 173.

[7] Swanson, M. B.; Ivancic, W. A.; Saxena, A. M.; Allton, J. D.;
O’Brien, G. K.; Suzuki, T.; Nishizawa, H.; Nokata, M. J. Agric. Food Chem. 1995, 43, 513.

[8] Lahm, G. P., Selby, T. P.; Freudenberger, J. H.; Stevenson, T. M.; Myers, B. J.; Seburyamo, G.; Smith, B. K; Flexner, L.; Clark, C. E.; Cordova, D. Bioorg. Med. Chem. Lett. 2005, 15, 4898.

[9] Penning, T. D.; Talley, J. J.; Bertenshaw, S. R.; Carter, J. S.; Collins, P. W.; Docter, S.; Graneto, M. J.; Lee, L. F.; Malecha, J. W.; Miyashiro, J. M.; Rogers, R. S.; Rogier, D. J.; Yu, S. S.; Anderson, G. D.; Burton, E. G.; Cogburn, J. N.; Gregory, S. A.; Koboldt, C. M.; Perkins, W. E. Seibert, K.; Veenhuizen, A. W.; Zhang, Y. Y.; Isakson, P. C. J. Med. Chem. 1997, 40, 1347.

[10] Teng, M.; Zhu, J. J.; Johnson, M. D.; Chen, P.; Kornmann, J.; Chen, E. T.; Blasina, A.; Register, J.; Anderes, K.; Rogers, C.; Deng, Y. L.; Ninkovic, S.; Grant, S.; Hu, Q. Y.; Lundgren, K.; Peng, Z. W.; Kania, R. S. J. Med. Chem. 2007, 50, 5253.

[11] Ouyang, G. P.; Cai, X. J.; Chen, Z.; Song, B. A.; Bhadury, P. S.; Yang, S.; Jin, L. H.; Xue, W.; Hu, D. Y.; Zeng, S. J. Agric. Food Chem. 2008, 56, 60.

[12] Dai, H.; Shi, L.; Zhang, H. J.; Li, Y. Q.; Fang, J. X.; Shi, Y. J. Chin. J. Org. Chem. 2012, 32, 1060 (in Chinese).

(戴红, 施否, 张海军, 李永强, 方建新, 石玉军, 有机化学, 2012, 32, 1060.)

[13] Wang, X.; Wang, C. Q.; Fu, C. R.; Zou, X. M. Chin. J. Org. Chem. 2015, 35, 92 (in Chinese). (王釒金, 王朝强, 傅翠蓉, 邹小毛, 有机化学, 2015, 35, 92.)

[14] Dai, H.; Zhuang. H. Y.; Shi, L.; Li, G.; Zhang, H. J.; Fang, Y.; Dai, B. J. Chin. J. Org. Chem. 2015, 35, 2399 (in Chinese). (戴红, 庄辉阳, 施否, 李刚, 张海军, 方源, 戴宝江, 有机化学, 2015, 35, 2399.)

[15] Tian, Z. Z.; Shao, X. S.; Li, Z.; Qian, X. H.; Huang, Q. C. J. Agric. Food Chem. 2007, 55, 2288.

[16] Lu, S. Y.; Shao, X. S.; Li, Z.; Xu, Z. P.; Zhao, S. S.; Wu, Y. L.; Xu, X. Y. J. Agric. Food Chem. 2012, 60, 322.

[17] Selby, T. P.; Lahm, G. P.; Stevenson, T. M.; Hughes, K. A.; Cordova, D.; Annan, I. B.; Barry, J. D.; Benner, E. A.; Currie, M. J.; Pahutski, T. F. Bioorg. Med. Chem. Lett. 2013, 23, 6341.

[18] Song, B. A.; Liu, X. H.; Yang, S.; Hu, D. Y.; Jin, L. H.; Zhang, Y. T. Chin. J. Org. Chem. 2005, 25, 507 (in Chinese).

(宋宝安, 刘新华, 杨松, 胡德禹, 金林红, 张玉涛, 有机化学, 2005, 25, 507.)

[19] Ouyang, G. P.; Chen, Z.; Cai, X. J.; Song, B. A.; Bhadury, P. S.; Yang, S.; Jin, L. H.; Xue, W.; Hu, D. Y., Zeng, S. Bioorg. Med. Chem. 2008, 16, 9699.

[20] Shi, Y. J.; Wang, S. L.; He, H. B.; Li, Y.; Li, Y.; Fang, Y.; Dai, H. Chin. J. Org. Chem. 2015, 35, 1785 (in Chinese).

(石玉军, 王森林, 何海兵, 李钰, 李阳, 方源, 戴红, 有机化学, 2015, 35, 1785.)

[21] Dai, H.; Li, H.; Jin. Z. C.; Liu, W. Y.; Xiao, Y.; He, H. B.; Wang, Q. M.; Shi, Y. J. Chin. J. Org. Chem. 2016, 36, 185 (in Chinese). (戴红, 李宏, 金智超, 刘文永, 肖瑶, 何海兵, 汪清民, 石玉军, 有机化学, 2016, 36, 185.)

[22] Song, H. J.; Liu, Y. X.; Xiong, L. X.; Li, Y. Q.; Yang, N.; Wang, Q. M. J. Agric. Food Chem. 2013, 61, 8730.

[23] Liu, X. H.; Cui, P.; Song, B. A.; Bhadury, P. S.; Zhu, H. L.; Wang, S. F. Bioorg. Med. Chem. 2008, 16, 4075.

[24] Park, M. S.; Park, H. J.; Park, K. H.; Lee, K. I. Synth. Commun. 2004, 34, 1541.

[25] Ma, J. A.; Huang, R. Q.; Feng, L.; Song, J.; Qiu, D. W. Chem. Res. Chin. Univ. 2003, 19, 297.

[26] Dai, H.; Xiao, Y. S.; Li, Z.; Xu, X. Y.; Qian, X. H. Chin. Chem. Lett. 2014, 25, 1014.

[27] Liu, J. C.; Liu, Y. J.; He, H. W. Chin. J. Org. Chem. 2015, 35, 462 (in Chinese). (刘建超, 刘勇军, 贺红武, 有机化学, 2015, 35, 462.)

[28] Song, B. A.; Yang, S.; Zhong, H. M.; Jin, L. H.; Hu, D. Y.; Liu, G. J. Fluorine Chem. 2015, 126, 87. 